\title{
Polaron physics in optical lattices
}

\author{
Martin Bruderer, Alexander Klein, Stephen R. Clark, and Dieter Jaksch \\ Clarendon Laboratory, University of Oxford, Parks Road, Oxford OX1 3PU, United Kingdom
}

(Received 6 March 2007; published 13 July 2007)

\begin{abstract}
We investigate the effects of a nearly uniform Bose-Einstein condensate (BEC) on the properties of immersed trapped impurity atoms. Using a weak-coupling expansion in the BEC-impurity interaction strength, we derive a model describing polarons, i.e., impurities dressed by a coherent state of Bogoliubov phonons, and apply it to ultracold bosonic atoms in an optical lattice. We show that, with increasing BEC temperature, the transport properties of the impurities change from coherent to diffusive. Furthermore, stable polaron clusters are formed via a phonon-mediated off-site attraction.
\end{abstract}

DOI: 10.1103/PhysRevA.76.011605 PACS number(s): 03.75.-b, 03.67.-a, 71.38.Mx, 71.38.Ht

The lack of lattice phonons is a distinguishing feature of optical lattices, i.e., conservative optical potentials formed by counterpropagating laser beams, and contributes to the excellent coherence properties of atoms trapped in them [1]. However, some of the most interesting phenomena in condensed matter physics involve phonons, and thus it is also desirable to introduce them in a controlled way into optical lattices. Recently, it has been shown that immersing an optical lattice into a Bose-Einstein condensate (BEC) leads to interband phonons, which can be used to load and cool atoms to extremely low temperatures [2]. Here, we instead concentrate on the dynamics within the lowest Bloch band of an immersed lattice, and show how intraband phonons lead to the formation of polarons $[3,4]$. This has a profound effect on lattice transport properties, inducing a crossover from coherent to incoherent hopping as the BEC temperature increases. Furthermore, polarons aggregate on adjacent lattice sites into stable clusters, which are not prone to loss from inelastic collisions. Since these phenomena are relevant to the physics of conduction in solids, introducing phonons into an optical lattice system may lead to a better understanding of hightemperature superconductivity $[3,5]$ and charge transport in organic molecules [6]. Additionally, this setup may allow the investigation of the dynamics of classically indistinguishable particles [7].

Experimental progress in trapping and cooling atoms has recently made a large class of interacting many-body quantum systems [8] accessible. For instance, the formation of repulsively bound atom pairs on a single site has been demonstrated [9], and strongly correlated mixtures of degenerate quantum gases have been realized [10]. In such Bose-Fermi mixtures, rich phase diagrams, including charge and spin density wave phases $[11,12]$, pairing of fermions with bosons [13], and a supersolid phase [14], can be expected. Here we instead consider one atomic species, denoted as the impurities, confined to a trapping potential, for example an optical lattice, immersed in a nearly uniform BEC, shown in Fig. 1. Based on a weak-coupling expansion in the BEC-impurity interaction strength, we derive a model in terms of polarons, which are composed of impurity atoms dressed by a coherent state of Bogoliubov phonons [3,4]. The model also includes attractive impurity-impurity interactions mediated by the phonons $[15,16]$. An essential requirement for our model is that neither interactions with impurities nor the trapping potential confining the impurities impairs the ability of the surrounding gas to sustain phononlike excitations. The first con- dition limits the number of impurity atoms [10], whereas the latter requirement can be met by using a species-specific optical lattice potential [17]. Moreover, unlike in the case of self-localized impurities [18], we assume that the oneparticle states of the impurities are not modified by the BEC, which can be achieved by sufficiently tight impurity trapping.

The Hamiltonian of the system is composed of three parts, $\hat{H}=\hat{H}_{\chi}+\hat{H}_{B}+\hat{H}_{I}$, where $\hat{H}_{\chi}$ governs the dynamics of the impurity atoms, which can be either bosonic or fermionic. The BEC Hamiltonian $\hat{H}_{B}$ and the density-density interaction Hamiltonian $\hat{H}_{I}$ are

$$
\begin{gathered}
\hat{H}_{B}=\int d \mathbf{r} \hat{\phi}^{\dagger}(\mathbf{r})\left(-\frac{\hbar^{2} \nabla^{2}}{2 m_{b}}+V_{\text {ext }}(\mathbf{r})+\frac{g}{2} \hat{\phi}^{\dagger}(\mathbf{r}) \hat{\phi}(\mathbf{r})\right) \hat{\phi}(\mathbf{r}), \\
\hat{H}_{I}=\kappa \int d \mathbf{r} \hat{\chi}^{\dagger}(\mathbf{r}) \hat{\chi}(\mathbf{r}) \hat{\phi}^{\dagger}(\mathbf{r}) \hat{\phi}(\mathbf{r}),
\end{gathered}
$$

where $\hat{\chi}(\mathbf{r})$ is the impurity field operator, and $\hat{\phi}(\mathbf{r})$ is the condensate atom field operator satisfying the commutation relations $\left[\hat{\phi}(\mathbf{r}), \hat{\phi}^{\dagger}\left(\mathbf{r}^{\prime}\right)\right]=\delta\left(\mathbf{r}-\mathbf{r}^{\prime}\right)$ and $\left[\hat{\phi}(\mathbf{r}), \hat{\phi}\left(\mathbf{r}^{\prime}\right)\right]=0$. The coupling constants $g>0$ and $\kappa$ account for the boson-boson and impurity-boson interaction, respectively, $m_{b}$ is the mass of a condensate atom, and $V_{\text {ext }}(\mathbf{r})$ is a weak external trapping potential. Without yet specifying $\hat{H}_{\chi}$, we expand $\hat{\chi}(\mathbf{r})$ $=\Sigma_{\nu} \eta_{\nu}(\mathbf{r}) \hat{a}_{\nu}$, where $\eta_{\nu}(\mathbf{r})$ are a set of orthogonal mode functions of the impurities, and $\hat{a}_{\nu}\left(\hat{a}_{\nu}^{\dagger}\right)$ the corresponding annihilation (creation) operators, labeled by the quantum numbers $\nu$.

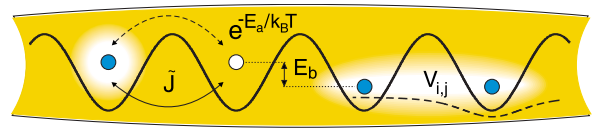

FIG. 1. (Color online) A quantum degenerate gas confined to an optical lattice is immersed in a much larger BEC. For increasing BEC temperature $T$, a crossover from coherent to diffusive hopping, characterized by $\widetilde{J}$ and $E_{a}$, respectively, can be observed. The phonon-induced interaction potential $V_{i, j}$ leads to the formation of off-site polaron clusters, separated by a gap $E_{b}$ from the continuum of unbound states. 
A common approach to find the elementary excitations of the BEC in the presence of impurities is to solve the GrossPitaevskii equation (GPE) [19] for the full system, i.e., for $\kappa \neq 0$, and to subsequently quantize small oscillations around the classical ground state. To obtain a quantum description of the impurity dynamics, we instead solve the GPE without taking $\hat{H}_{I}$ into account, and express the BEC deformations around the impurities as coherent states of Bogoliubov phonons. Specifically, we write $\hat{\phi}(\mathbf{r})=\phi_{0}(\mathbf{r})+\delta \hat{\phi}(\mathbf{r})$, with $\phi_{0}(\mathbf{r})=\phi_{0}^{*}(\mathbf{r})$ the solution of the GPE for $\kappa=0$. Provided that the impurity-boson coupling is sufficiently weak, i.e., $|\kappa| / g n_{0}(\mathbf{r}) \xi^{D}(\mathbf{r}) \ll 1$, with $\xi(\mathbf{r})=\hbar / \sqrt{m_{b} g n_{0}(\mathbf{r})}$ the healing length, $n_{0}(\mathbf{r})=\phi_{0}^{2}(\mathbf{r})$, and $D$ the number of spatial dimensions, we expect that the deviation of $\hat{\phi}(\mathbf{r})$ from $\phi_{0}(\mathbf{r})$ is of order $\kappa$, i.e., $\langle\delta \hat{\phi}(\mathbf{r})\rangle \propto \kappa$, where $\langle\cdot\rangle$ stands for the expectation value. We insert $\phi_{0}(\mathbf{r})+\delta \hat{\phi}(\mathbf{r})$ into the Hamiltonian $\hat{H}_{B}+\hat{H}_{I}$, keep terms up to second order in $\kappa$, and obtain the linear term $\kappa \int d \mathbf{r} \hat{\chi}^{\dagger}(\mathbf{r}) \hat{\chi}(\mathbf{r}) \phi_{0}(\mathbf{r})\left[\delta \hat{\phi}^{\dagger}(\mathbf{r})+\delta \hat{\phi}(\mathbf{r})\right]$, in addition to the standard constant and quadratic terms in $\delta \hat{\phi}(\mathbf{r})$ and $\delta \hat{\phi}^{\dagger}(\mathbf{r})$, since $\phi_{0}(\mathbf{r})$ is no longer the ground state of the system.

In order to diagonalize the quadratic terms in $\delta \hat{\phi}(\mathbf{r})$ and $\delta \hat{\phi}^{\dagger}(\mathbf{r})$, we use the expansion $\delta \hat{\phi}(\mathbf{r})=\Sigma_{\mu}\left[u_{\mu}(\mathbf{r}) \hat{b}_{\mu}-v_{\mu}^{*}(\mathbf{r}) \hat{b}_{\mu}^{\dagger}\right]$, where $u_{\mu}(\mathbf{r})$ and $v_{\mu}^{*}(\mathbf{r})$ are the solutions of the Bogoliubov-de Gennes equations [19] for $\kappa=0$, and $\hat{b}_{\mu}\left(\hat{b}_{\mu}^{\dagger}\right)$ are the bosonic Bogoliubov annihilation (creation) operators, labeled by the quantum numbers $\mu$. We assume that the mode functions $\eta_{\nu}(\mathbf{r})$ are localized on a length scale much smaller than is set by $V_{\text {ext }}(\mathbf{r})$, and that $\int d \mathbf{r}\left|\eta_{\nu}(\mathbf{r})\right|^{2}\left|\eta_{\tau}(\mathbf{r})\right|^{2} \approx 0$ for $\nu \neq \tau$, i.e., the probability densities $\left|\eta_{\nu}(\mathbf{r})\right|^{2}$ for different mode functions deviate appreciably from zero only within mutually exclusive spatial regions. In this case, $\int d \mathbf{r} \phi_{0}(\mathbf{r})\left[u_{\mu}(\mathbf{r})-v_{\mu}(\mathbf{r})\right] \eta_{\nu}(\mathbf{r}) \eta_{\tau}^{*}(\mathbf{r}) \approx 0$ and $\int d \mathbf{r} n_{0}(\mathbf{r}) \eta_{\nu}(\mathbf{r}) \eta_{\tau}^{*}(\mathbf{r}) \approx 0$ hold for $\nu \neq \tau$, and hence the nondiagonal impurity-phonon coupling is negligible. The total Hamiltonian can thus be rewritten in the form of a HubbardHolstein model [20] $\hat{H}=\hat{H}_{\chi}+\Sigma_{\nu, \mu} \hbar \omega_{\mu}\left(M_{\nu, \mu} \hat{b}_{\mu}+M_{\nu, \mu}^{*} \hat{b}_{\mu}^{\dagger}\right) \hat{n}_{\nu}$ $+\Sigma_{\nu} \bar{E}_{\nu} \hat{n}_{\nu}+\Sigma_{\mu} \hbar \omega_{\mu} \hat{b}_{\mu}^{\dagger} \hat{b}_{\mu}$, with $\hbar \omega_{\mu}$ the energies of the Bogoliubov excitations, the number operator $\hat{n}_{\nu}=\hat{a}_{\nu}^{\dagger} \hat{a}_{\nu}$, the dimensionless matrix elements $M_{\nu, \mu}=\left(\kappa / \hbar \omega_{\mu}\right) \int d \mathbf{r} \phi_{0}(\mathbf{r})$ $\times\left[u_{\mu}(\mathbf{r})-v_{\mu}(\mathbf{r})\right]\left|\eta_{\nu}(\mathbf{r})\right|^{2}$, and the mean field shift $\bar{E}_{\nu}$ $=\kappa \int d \mathbf{r} n_{0}(\mathbf{r})\left|\eta_{\nu}(\mathbf{r})\right|^{2}$. We obtain an effective Hamiltonian $\hat{H}_{\text {eff }}$ including corrections to $\phi_{0}(x)$ of order $\kappa$ by applying the unitary Lang-Firsov transformation $[3,4] \hat{H}_{\text {eff }}=\hat{U} \hat{H} \hat{U}^{\dagger}$, with $\hat{U}=\exp \left[\Sigma_{\nu, \mu}\left(M_{\nu, \mu}^{*} \hat{b}_{\mu}^{\dagger}-M_{\nu, \mu} \hat{b}_{\mu}\right) \hat{n}_{\nu}\right]$, which yields

$$
\begin{aligned}
\hat{H}_{\mathrm{eff}}= & \hat{U} \hat{H}_{\chi} \hat{U}^{\dagger}+\sum_{\nu}\left(\bar{E}_{\nu}-E_{\nu}\right) \hat{n}_{\nu}-\sum_{\nu} E_{\nu} \hat{n}_{\nu}\left(\hat{n}_{\nu}-1\right) \\
& -\frac{1}{2} \sum_{\nu \neq \tau} V_{\nu, \tau} \hat{n}_{\nu} \hat{n}_{\tau}+\sum_{\mu} \hbar \omega_{\mu} \hat{b}_{\mu}^{\dagger} \hat{b}_{\mu} .
\end{aligned}
$$

The transformed impurity Hamiltonian $\hat{U} \hat{H}_{\chi} \hat{U}^{\dagger}$ is obtained using the relation $\hat{U} \hat{a}_{\nu}^{\dagger} \hat{U}^{\dagger}=\hat{a}_{\nu}^{\dagger} \hat{X}_{\nu}^{\dagger}$, where $\hat{X}_{\nu}^{\dagger}=\exp \left[\Sigma_{\mu}\left(M_{\nu, \mu}^{*} \hat{b}_{\mu}^{\dagger}\right.\right.$ $\left.\left.-M_{\nu, \mu} \hat{b}_{\mu}\right)\right]$ is a Glauber displacement operator that creates a coherent phonon cloud, i.e., a BEC deformation, around the impurity. In the limit where the BEC adjusts instantaneously to the impurity configuration, polarons created by $\hat{a}_{\nu}^{\dagger} \hat{X}_{\nu}^{\dagger}$ are the appropriate quasiparticles, and $\hat{H}_{\text {eff }}$ describes a nonretarded interaction with the potential $V_{\nu, \tau}=\Sigma_{\mu} \hbar \omega_{\mu}\left(M_{\nu, \mu} M_{\tau, \mu}^{*}\right.$ $\left.+M_{\nu, \mu}^{*} M_{\tau, \mu}\right)$. The polaronic level shift $E_{\nu}=\Sigma_{\mu} \hbar \omega_{\mu}\left|M_{\nu, \mu}\right|^{2}$ is equal to the characteristic potential energy of an impurity in the deformed BEC.

We now turn to the specific case of bosons loaded into an optical lattice immersed in a homogeneous BEC [2]. In the tight-binding approximation, the impurity dynamics is well described by the Bose-Hubbard model $\hat{H}_{\chi}=-J \Sigma_{\langle i, j\rangle} \hat{a}_{i}^{\dagger} \hat{a}_{j}$ $+(U / 2) \sum_{j} \hat{n}_{j}\left(\hat{n}_{j}-1\right)+\mu \sum_{j} \hat{n}_{j}$, where $\mu$ describes the energy offset, $U$ the on-site interaction strength, and $J$ the hopping matrix element between adjacent sites [8,21]. The modes of the lattice atoms are Wannier functions $\eta_{j}(\mathbf{r})$ of the lowest Bloch band localized at site $j$, and $\langle i, j\rangle$ denotes the sum over nearest neighbors. Noting that $\left[\hat{U}, \hat{n}_{j}\right]=0$, we find

$$
\hat{U} \hat{H}_{\chi} \hat{U}^{\dagger}=-J \sum_{\langle i, j\rangle}\left(\hat{X}_{i} \hat{a}_{i}\right)^{\dagger} \hat{X}_{j} \hat{a}_{j}+\frac{1}{2} U \sum_{j} \hat{n}_{j}\left(\hat{n}_{j}-1\right)+\mu \sum_{j} \hat{n}_{j},
$$

with the corresponding matrix elements $M_{j, \mathbf{q}}$ $=\kappa \sqrt{n_{0} \varepsilon_{\mathbf{q}} /\left(\hbar \omega_{\mathbf{q}}\right)^{3}} f_{j}(\mathbf{q})$, where $\mathbf{q}$ is the phonon momentum, $\varepsilon_{\mathbf{q}}=(\hbar \mathbf{q})^{2} / 2 m_{b}$ the free particle energy, $\hbar \omega_{\mathbf{q}}$ $=\sqrt{\varepsilon_{\mathbf{q}}\left(\varepsilon_{\mathbf{q}}+2 g n_{0}\right)}$ the Bogoliubov dispersion relation, and $f_{j}(\mathbf{q})=\Omega^{-1 / 2} \int d \mathbf{r}\left|\eta_{j}(\mathbf{r})\right|^{2} \exp (i \mathbf{q} \cdot \mathbf{r})$, with $\Omega$ the quantization volume. We note that, for $|\mathbf{q}| \ll 1 / \xi$, we have $M_{j, \mathbf{q}}$ $\propto f_{j}(\mathbf{q}) / \sqrt{|\mathbf{q}|}$, whereas, for $|\mathbf{q}| \gg 1 / \xi$, one obtains $M_{j, \mathbf{q}}$ $\propto f_{j}(\mathbf{q}) / \mathbf{q}^{2}$.

The Hamiltonian $\hat{H}_{\text {eff }}$ describes the dynamics of hopping polarons according to an extended Hubbard model [8], provided that $c \gg a J / \hbar$, with $c \sim \sqrt{g n_{0} / m_{b}}$ the phonon velocity and $a$ the lattice spacing. We gain qualitative insight into the dependence of $V_{i, j}$ and the constant polaronic level shift $E_{\nu}$ $\equiv E_{p}$ on the system parameters by considering a onedimensional quasi-BEC in the thermodynamic limit. We assume a sufficiently deep lattice to approximate the Wannier functions by Gaussians of width $\sigma \ll \xi$, and find $V_{i, j}$ $=\left(\kappa^{2} / \xi g\right) \mathrm{e}^{-2|i-j| a / \xi}$ and $E_{p}=\kappa^{2} / 2 \xi g$. We note that the interaction between impurities is always attractive. More importantly, for realistic experimental parameters, $\xi \sim a$, and hence the off-site terms $V_{j, j+1} \propto e^{-2 a / \xi}$ are non-negligible. This interaction potential is a direct consequence of the local deformation of the BEC around each impurity, as shown in Fig. 1. For a set of static impurities at positions $x_{j}=a j$, the overall deformation of the BEC density to order $\kappa$ is given by $n(x)$ $=n_{0}+\sum_{j, \mathbf{q}}\left\langle\hat{X}_{j} \hat{b}_{\mathbf{q}}^{\dagger} \hat{b}_{\mathbf{q}} \hat{X}_{j}^{\dagger}\right\rangle=n_{0}-(\kappa / g \xi) \sum_{j} e^{-2 \mid x-x_{j} / \xi}$.

We first consider coherent hopping of polarons at small BEC temperatures $k_{B} T \ll E_{p}$, where incoherent phonon scattering is highly suppressed. Provided that $\zeta=J / E_{p} \ll 1$, we can apply the so-called strong-coupling theory [5], and treat the hopping term in Eq. (2) as a perturbation. Including terms of first order in $\zeta$, we obtain the impurity Hamiltonian 


$$
\hat{H}^{(1)}=-\widetilde{J} \sum_{\langle i, j\rangle} \hat{a}_{i}^{\dagger} \hat{a}_{j}+\tilde{\mu} \sum_{j} \hat{n}_{j}+\frac{1}{2} \widetilde{U} \sum_{j} \hat{n}_{j}\left(\hat{n}_{j}-1\right)-\frac{1}{2} \sum_{i \neq j} V_{i, j} \hat{n}_{i} \hat{n}_{j},
$$

with $\tilde{\mu}=\mu+\kappa n_{0}-E_{p}, \tilde{U}=U-2 E_{p}$, and $\tilde{J}=J\left\langle\left\langle\hat{X}_{i}^{\dagger} \hat{X}_{j}\right\rangle\right\rangle$. Here $\langle\langle\cdot\rangle\rangle$ denotes the average over the thermal phonon distribution and $i, j$ are nearest neighbors. We find $\left\langle\left\langle\hat{X}_{i}^{\dagger} \hat{X}_{j}\right\rangle\right\rangle$ $=\exp \left\{-\Sigma_{\mathbf{q} \neq \mathbf{0}}\left|M_{0, \mathbf{q}}\right|^{2}[1-\cos (\mathbf{q} \cdot \mathbf{a})]\left(2 N_{\mathbf{q}}+1\right)\right\}$, with $\mathbf{a}$ the position vector connecting two nearest neighbor sites and $N_{\mathbf{q}}=\left(e^{\hbar \omega_{\mathbf{q}} / k_{B} T}-1\right)^{-1}$. Thus, the hopping bandwidth decreases exponentially with increasing coupling constant $\kappa$ and temperature $T$.

At high temperatures $E_{p} \ll k_{B} T \ll k_{B} T_{c}$ (with $T_{c}$ the critical temperature of the BEC) inelastic scattering, in which phonons are emitted and absorbed, becomes dominant, and thus the transport of atoms through the lattice changes from being purely coherent to incoherent. We investigate this crossover by deriving a generalized master equation (GME) for a single particle starting from $\hat{H}_{\text {eff }}$ in Eq. (1). Using the Nakajima-Zwanzig projection method [22], we find that the occupation probabilities $P_{l}(t)$ at site $l$ and time $t$ evolve according to the GME [23]

$$
\frac{\partial P_{i}(t)}{\partial t}=\int_{0}^{t} d s \sum_{j}\left[W_{i, j}(s) P_{j}(t-s)-W_{j, i}(s) P_{i}(t-s)\right],
$$

where the effect of the phonon bath is encoded in the memory functions $W_{i, j}(s)$, which are symmetric in $(i, j)$. To first order in $\zeta$, thus keeping only nearest-neighbor correlations, we find

$$
\begin{aligned}
W_{i, j}(s)= & 2\left(\frac{J}{\hbar}\right)^{2} \operatorname{Re}\left[\operatorname { e x p } \left(2 \sum_{\mathbf{q} \neq 0}\left|M_{0, \mathbf{q}}\right|^{2}[1-\cos (\mathbf{q} \cdot \mathbf{a})]\right.\right. \\
& \left.\left.\times\left[\left(N_{\mathbf{q}}+1\right)\left(e^{i \omega_{\mathbf{q}} s}-1\right)+N_{\mathbf{q}}\left(e^{-i \omega_{\mathbf{q}} s}-1\right)\right]\right)\right],
\end{aligned}
$$

and $W_{i, j}(s)=0$ if $i$ and $j$ are beyond nearest neighbors. The nontrivial part of $W_{i, j}(s)$ takes the values $2(J / \hbar)^{2}$ at $s=0$ and $2(\widetilde{J} / \hbar)^{2}$ in the limit $s \rightarrow \infty$. In the regime $k_{B} T \ll E_{p}$, we have $\tilde{J} \sim J$, and the memory function $W_{i, j}(s)$ is well approximated by $2(\widetilde{J} / \hbar)^{2} \Theta(s)$ [with $\Theta(s)$ the Heaviside step function], which describes purely coherent hopping in agreement with $\hat{H}^{(1)}$. For $E_{p} \ll k_{B} T \ll k_{B} T_{c}$, we observe that $\widetilde{J} \ll J$, and $W_{i, j}(s)$ drops off sufficiently fast for the Markov approximation to be valid, as illustrated in Fig. 2(a). In this case one can replace $P_{l}(t-s)$ by $P_{l}(t)$ in Eq. (4) and after integration over $s$ the GME reduces to a standard Pauli master equation $\partial_{t} P_{i}(t)=\sum_{j} w_{i, j}\left[P_{j}(t)-P_{i}(t)\right]$ describing purely incoherent hopping. The hopping rate is of the form $w_{i, j}$ $\sim J^{2} \exp \left(-E_{a} / k_{B} T\right) /\left(\hbar \sqrt{k_{B} T E_{a}}\right)[4,20]$, where $E_{a} \sim E_{p}$ is the activation energy.

The temperature-dependent crossover from coherent to diffusive hopping in a quasi-one-dimensional (1D) system is apparent in the evolution for a time $\tau \sim 1 / w_{i, j}$ of a particle initially localized at lattice site $j=0$. The mean-squared displacement of the lattice atom, $\bar{l}^{2}(t)=\sum_{l} l^{2} P_{l}(t)$, can be decom-
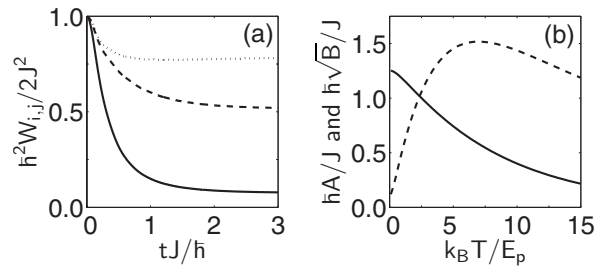

FIG. 2. (a) Memory function $\hbar^{2} W_{i, j}(t) /\left(2 J^{2}\right)$ versus time for $k_{B} T=0$ (dotted line), $2 E_{p}$ (dashed line), and $10 E_{p}$ (full line). It drops off rapidly for $k_{B} T \gg E_{p}$, indicating the dominance of incoherent hopping. (b) Coefficients $\hbar A / J$ (dashed line) and $\hbar \sqrt{B} / J$ (full line) versus temperature, obtained from the numerical solution of the GME in Eq. (4) for an evolution time $\tau=10 \hbar / J \approx 0.8 \times 10^{-3} \mathrm{~s}$. The condition $A=\sqrt{B}$ is satisfied at $k_{B} T / E_{p} \approx 2.3$, with $E_{p} / k_{B} \approx 11 \mathrm{nK}$. The lattice (wavelength $\lambda=790 \mathrm{~nm}$ ) contains a single ${ }^{41} \mathrm{~K}$ atom with $J=2.45 \times 10^{-2} E_{R}, \kappa / E_{R} \lambda=2.3 \times 10^{-2}$, the recoil energy $E_{R}=(2 \pi \hbar)^{2} / 2 m_{a} \lambda^{2}$, and $m_{a}$ the mass of the lattice atom. The BEC consists of ${ }^{87} \mathrm{Rb}$ atoms with $n_{0}=5 \times 10^{6} \mathrm{~m}^{-1}$ and $\mathrm{g} / E_{R} \lambda$ $=8.9 \times 10^{-3}$.

posed as $\overline{l^{2}}(t)=A t+B t^{2}$ into incoherent and coherent contributions, characterized by the coefficients $A$ and $B$, respectively. The crossover takes place when incoherent and coherent contributions to $\overline{l^{2}}(\tau)$ are comparable, i.e., $\sqrt{B}=A$. Figure 2(b) shows $A$ and $\sqrt{B}$ as functions of $T$, where $\overline{l^{2}}(t)$ was obtained by numerically solving the GME using the memory function $W_{i, j}$ in Eq. (5). We find that, for a ${ }^{41} \mathrm{~K}-{ }^{87} \mathrm{Rb}$ system [24] under standard experimental conditions, the crossover takes place well below the critical temperature of the BEC (see caption of Fig. 2).

We now discuss the formation of polaron clusters for $k_{B} T \lesssim E_{p}$, based on Hamiltonian $\hat{H}^{(1)}$ in Eq. (3), and assume that the bosonic impurities are in thermal equilibrium with the BEC. At these temperatures, $V_{i, j}$ and $\widetilde{J}$ are well approximated by their $T=0$ values. We consider the limit $\tilde{U} \gg V_{j, j+1}$, $\widetilde{U} \gg \widetilde{J}$, and adiabatically eliminate configurations with multiply occupied sites. Keeping only nearest neighbor interactions, we obtain approximate expressions for the binding energy $E_{b}(s) \approx(s-1) V_{j, j+1}$ of a cluster of $s$ polarons located in adjacent sites and the lowest energy band $E_{k}(s) \approx-E_{b}(s)$ $-2 \widetilde{J}^{s}\left(V_{j, j+1}\right)^{1-s} \cos (k a)$ [25], with $k$ the quasimomentum. This band approximation is in good agreement with the results from exact diagonalization of $\hat{H}^{(1)}$ using the full interaction potential $V_{i, j}$, as shown for three polarons in Fig. 3(a).

This model predicts a decreasing average cluster size with increasing temperature. For a small system with $N=3$ polarons, we calculate the probability of finding a three-polaron cluster $P_{3}=\Sigma_{\nu}\left\langle n_{\nu}\right\rangle$, where the sum is taken over all states with energies $\varepsilon_{\nu}<E_{g}(2)+E_{g}(1), E_{g}(N)$ is the ground state energy of an $N$-polaron cluster, and the occupation probabilities are given by the Boltzmann law $\left\langle n_{\nu}\right\rangle \propto \exp \left(-\varepsilon_{\nu} / k_{B} T\right)$. Analogously, we determine the probability $P_{2}$ of finding a two-polaron cluster or bipolaron. The results are shown in Fig. 3(b). The probability of having a three-polaron cluster goes down with increasing temperature, and for $T=E_{p} / k_{B}$ $\approx 18 \mathrm{nK}$ is essentially zero for the parameters chosen. For this three-polaron cluster, three-particle loss is negligible due to the on-site repulsion $U$. Decreasing the value of $U$ gives a 


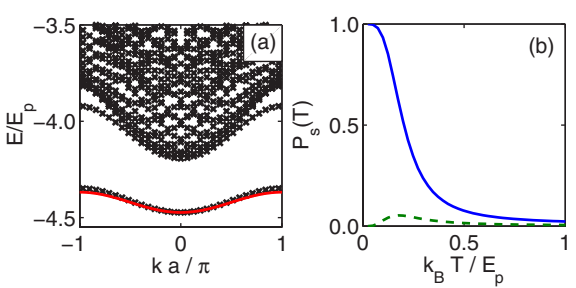

FIG. 3. (Color online) (a) Energy spectrum of three polarons in a 1D optical lattice. The solid line shows the lowest energy band $E_{3}(k)$ characterizing off-site three-polaron clusters. The lattice (wavelength $\lambda=790 \mathrm{~nm}, M=31$ sites, periodic boundary conditions) contains ${ }^{133} \mathrm{Cs}$ atoms with $\widetilde{J}=7.5 \times 10^{-3} E_{R}, U=50 E_{p}$, and $\kappa / E_{R} \lambda=1.05 \times 10^{-1}$. The BEC consists of ${ }^{87} \mathrm{Rb}$ atoms with $n_{0}=5 \times 10^{6} \mathrm{~m}^{-1}$ and $g / E_{R} \lambda=4.5 \times 10^{-2}$. (b) Probability of finding a three-polaron cluster (solid line) or a two-polaron cluster (dashed line) versus temperature. The parameters are $g / E_{R} \lambda=6.5 \times 10^{-2}$, $\kappa / E_{R} \lambda=1.32 \times 10^{-1}, U=2.2 E_{p}, M=27$, and the rest as for (a).

significantly increased $P_{2}$ compared to the values shown in Fig. 3(b).

The clustering of polarons leads to their mutual exponential localization. This is illustrated by the density-density correlations $\left\langle\hat{n}_{i} \hat{n}_{i+j}\right\rangle$ for the three-polaron cluster in Fig. 4(a). With increasing attractive interaction $V_{i, j}$, the mutual localization gets stronger, leading to an increased broadening of the momentum distribution, as shown in Fig. 4(b). This allows polaron clusters to be identified in time of flight experiments. We note that the transition from a superfluid to a Mott insulator also leads to broadening of the momentum distribution as, e.g., observed in [10]. However, using Bragg spectroscopy [9] would allow the unambiguous distinction of boson clustering from this transition.
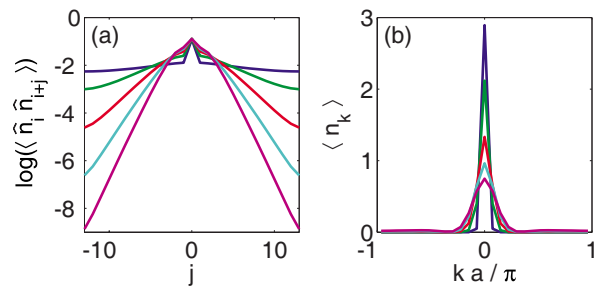

FIG. 4. (Color online) (a) Density-density correlation in a system of three polarons indicating the formation of off-site threepolaron clusters. (b) Corresponding momentum distribution $\left\langle n_{k}\right\rangle$. $\kappa / E_{R} \lambda=\{4.0,6.1,8.1,10.1,12.1\} \times 10^{-2}$ where a higher value corresponds to a more localized correlation in (a) and a more spread distribution in (b). The other parameters are $g / E_{R} \lambda=6.5 \times 10^{-2}$, $M=27, U=3 E_{p}$, and the rest as in Fig. 3(a).

We have demonstrated that the dynamics of bosonic impurities immersed in a BEC is accurately described in terms of polarons. We found that spatial coherence is destroyed in hopping processes at large BEC temperatures, while the main effect of the BEC at low temperatures is to reduce the coherent hopping rate. Furthermore, the phonons induce offsite interactions leading to the formation of stable clusters, which are not affected by loss due to inelastic collisions. Using the techniques introduced in this paper, qualitatively similar phenomena can also be shown to occur for fermionic impurities. In either case these effects can be controlled by external parameters and lie within the reach of current experimental techniques.

M.B. thanks Danail Obreschkow for fruitful discussions. This work was supported by the U.K. EPSRC through QIP IRC (Grant No. GR/S82176/01) and Project No. EP/ C51933/1, the EU through the STREP project OLAQUI, the Berrow Society (M.B.), and the Keble Association (A.K.).
[1] I. Bloch, Nat. Phys. 1, 23 (2005).

[2] A. Griessner, A. J. Daley, S. R. Clark, D. Jaksch, and P. Zoller, Phys. Rev. Lett. 97, 220403 (2006).

[3] A. S. Alexandrov and N. F. Mott, Rep. Prog. Phys. 57, 1197 (1994).

[4] G. M. Mahan, Many-Particle Physics, 3rd ed. (Kluwer Academic, New York, 2000).

[5] A. S. Alexandrov, J. Ranninger, and S. Robaszkiewicz, Phys. Rev. B 33, 4526 (1986).

[6] P. T. Henderson, D. Jones, G. Hampikian, Y. Kan, and G. B. Schuster, Proc. Natl. Acad. Sci. U.S.A. 96, 8353 (1999).

[7] D. Gottesman, e-print arXiv:cond-mat/0511207.

[8] M. Lewenstein, A. Sanpera, V. Ahufinger, B. Damski, A. Sen, and U. Sen, Adv. Phys. 56, 243 (2007).

[9] K. Winkler et al., Nature (London) 441, 853 (2006).

[10] K. Günter, T. Stöferle, H. Moritz, M. Köhl, and T. Esslinger, Phys. Rev. Lett. 96, 180402 (2006); S. Ospelkaus et al., ibid. 96, 180403 (2006).

[11] L. Mathey, D.-W. Wang, W. Hofstetter, M. D. Lukin, and E. Demler, Phys. Rev. Lett. 93, 120404 (2004).

[12] E. Pazy and A. Vardi, Phys. Rev. A 72, 033609 (2005).

[13] M. Lewenstein, L. Santos, M. A. Baranov, and H. Fehrmann, Phys. Rev. Lett. 92, 050401 (2004).
[14] H. P. Büchler and G. Blatter, Phys. Rev. Lett. 91, 130404 (2003).

[15] J. Bardeen, G. Baym, and D. Pines, Phys. Rev. 156, 207 (1967).

[16] A. Klein and M. Fleischhauer, Phys. Rev. A 71, 033605 (2005).

[17] L. J. LeBlanc and J. H. Thywissen, Phys. Rev. A 75, 053612 (2007).

[18] K. Sacha and E. Timmermans, Phys. Rev. A 73, 063604 (2006).

[19] P. Öhberg et al., Phys. Rev. A 56, R3346 (1997).

[20] T. Holstein, Ann. Phys. (N.Y.) 8, 343 (1959).

[21] D. Jaksch, C. Bruder, J. I. Cirac, C. W. Gardiner, and P. Zoller, Phys. Rev. Lett. 81, 3108 (1998); D. Jaksch and P. Zoller, Ann. Phys. (N.Y.) 315, 52 (2005).

[22] H.-P. Breuer and F. Petruccione, The Theory of Open Quantum Systems (Oxford University Press, Oxford, 2002).

[23] V. M. Kenkre, Phys. Rev. B 12, 2150 (1975).

[24] G. Modugno, M. Modugno, F. Riboli, G. Roati, and M. Inguscio, Phys. Rev. Lett. 89, 190404 (2002).

[25] A. C. Scott, J. C. Eilbeck, and H. Gilhøj, Physica D 78, 194 (1994). 